\title{
The Emergent Flux and Effective Temperature of $\delta$ Canis Majoris
}

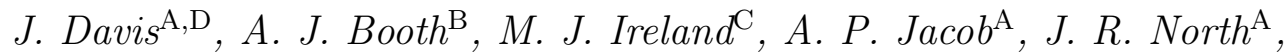 \\ S. M. Owens ${ }^{\mathrm{A}}$, J. G. Robertson ${ }^{\mathrm{A}}$, W. J. Tango ${ }^{\mathrm{A}}$ and P. G. Tuthill ${ }^{\mathrm{A}}$ \\ A School of Physics, University of Sydney, NSW 2006, Australia \\ B Jet Propulsion laboratory, California Institute of Technology, Pasadena, CA 91109, USA \\ ${ }^{\mathrm{C}}$ Planetary Science, MS 150-21, Caltech, 1200 E. California Blvd, Pasadena, CA 91125, USA \\ D Email: j.davis@physics.usyd.edu.au
}

\begin{abstract}
New angular diameter determinations for the bright southern F8 supergiant $\delta$ CMa enable the bolometric emergent flux and effective temperature of the star to be determined with improved accuracy. The spectral flux distribution and bolometric flux have been determined from published photometry and spectrophotometry and combined with the angular diameter to derive the bolometric emergent flux $\mathcal{F}=(6.50 \pm 0.24) \times 10^{7} \mathrm{Wm}^{-2}$ and the effective temperature $T_{\text {eff }}=5818 \pm 53 \mathrm{~K}$. The new value for the effective temperature is compared with previous interferometric and infrared flux method determinations. The accuracy of the effective temperature is now limited by the uncertainty in the bolometric flux rather than by the uncertainty in the angular diameter.
\end{abstract}

Keywords: stars: atmospheres-stars: fundamental parameters-stars: individual $(\delta \mathrm{CMa})$-techniques: interferometric

\section{Introduction}

In the determination of 32 stellar effective temperatures by Code et al. (1976), which is still the basis of the temperature scale for hot stars, the coolest and faintest star, and the star with the largest temperature uncertainty $( \pm 7 \%)$ was the southern F8 supergiant $\delta$ CMa (HR2693, HD54605). The effective temperatures were determined by combining the angular diameters measured with the Narrabri Stellar Intensity Interferometer (NSII) Hanburv Brown. Davis \& Allen 1974) with flux distributions constructed from various sources of calibrated photometry and spectrophotometry. The angular diameter of $\delta$ CMa determined with the NSII at a wavelength of $443 \mathrm{~nm}$ had an uncertainty of $\pm 14 \%$ and this was the dominant uncertainty in the effective temperature determination. Because the angular diameter was the least accurately determined with the NSII it has been a prime target for the Sydney University Stellar Interferometer (SUSI) (Davis et al. 1999a) as a demonstration of the improvement achieved in angular diameter measurements. The angular diameter has been measured with SUSI at wavelengths of $442 \mathrm{~nm}$ (Davis et al. 1999b) and $700 \mathrm{~nm}$ (Davis et al. 2007 ) with greatly improved accuracy. In this paper we use the angular diameter with revised fluxes obtained from published photometry and spectrophotometry to determine the bolometric emergent flux and the effective temperature for $\delta \mathrm{CMa}$ with significantly improved accuracy. The accuracy is now limited by the uncertainty in the determination of the bolometric flux received from the star after correction for interstellar extinction. The new directly determined temperature is also compared with the effective temperature determined by the infra-red flux method (IRFM).

The emergent flux at the surface of a star per unit wavelength interval $\left(\mathcal{F}_{\lambda}\right)$ is given by

$$
\mathcal{F}_{\lambda}=\frac{4}{\theta_{\mathrm{LD}}^{2}} f_{\lambda}
$$

where $\theta_{\mathrm{LD}}$ is the true limb-darkened angular diameter of the star and $f_{\lambda}$ is the flux per unit wavelength interval received at the Earth from the star at wavelength $\lambda$, corrected for atmospheric and interstellar extinction. The effective temperature of the star $\left(T_{\text {eff }}\right)$ is then given by

$$
\sigma T_{\text {eff }}^{4}=\mathcal{F}=\int_{0}^{\infty} \mathcal{F}_{\lambda} d \lambda=\frac{4}{\theta_{\mathrm{LD}}^{2}} \int_{0}^{\infty} f_{\lambda} d \lambda
$$

where $\sigma$ is the Stefan-Boltzmann radiation constant and $\mathcal{F}$ is the bolometric emergent flux at the stellar surface.

Thus, a knowledge of the limb-darkened angular diameter of the star, and the flux distribution received from it, leads to a direct determination of $T_{\text {eff }} . f_{\lambda}$ can be obtained from flux-calibrated photometry and spectrophotometry, and $\theta_{\text {LD }}$ can be obtained by interferometric measurements. In the following sections we will consider the determination of these two quantities for $\delta \mathrm{CMa}$, and finally their combination to give $\mathcal{F}$ and $T_{\text {eff }}$.

\section{The Angular Diameter}

The values for the equivalent uniform-disk angular diameter, $\theta_{\text {UD }}$, determined with the NSII and with SUSI have been discussed by Davis et al. (2007). The values for $\theta_{\text {UD }}$ determined with the NSII and at $695.6 \mathrm{~nm}$ with SUSI, taken from their Table 3, are listed in Table 1. The SUSI values in Table 1 for $442 \mathrm{~nm}$ are the revised values discussed by Davis et al. (2007) obtained after 
Table 1: The uniform-disk angular diameter of $\delta$ CMa determined with the NSII and with SUSI. $\lambda$ is the effective wavelength and $\Delta \lambda$ the spectral bandwidth of the measurement. $V_{0}^{2}$ is the extrapolated value of visibility squared at zero baseline from the uniform-disk angular diameter fit to the observed values of $V^{2} . \sigma \%$ is the percentage uncertainty in the uniform-disk angular diameter. The SUSI values for $442 \mathrm{~nm}$ are revised values from a re-processing of the original data (see text).

\begin{tabular}{cccccc}
\hline Instrument & $\begin{array}{c}\lambda \\
(\mathrm{nm})\end{array}$ & $\begin{array}{c}\Delta \lambda \\
(\mathrm{nm})\end{array}$ & $V_{0}^{2}$ & $\begin{array}{c}\theta_{\mathrm{UD}} \\
(\mathrm{mas})\end{array}$ & $\sigma \%$ \\
\hline NSII & 443.0 & 8 & $0.93 \pm 0.18$ & $3.29 \pm 0.46$ & 14.0 \\
SUSI & 442.0 & 4 & $0.917 \pm 0.024$ & $3.41 \pm 0.10$ & 2.6 \\
SUSI & 442.0 & 4 & $0.880 \pm 0.031$ & $3.37 \pm 0.15$ & 4.5 \\
SUSI & 696.6 & 80 & $1.003 \pm 0.012$ & $3.457 \pm 0.024$ & 0.7 \\
\hline
\end{tabular}

re-processing the observational data with the omission of observations not bracketed by a calibrator. All the determinations involved a two-parameter fit to the measured values of the square of the fringe visibility $\left(V^{2}\right)$, the fitting parameters being the equivalent uniform-disk angular diameter and the value of $V^{2}$ at zero baseline, $V_{0}^{2}$. The values of $V_{0}^{2}$ for each of the fits are included in Table 1.

Davis et al. (2007) determined, for each value of the uniform-disk angular diameter, the true, limb-darkened angular diameter of $\delta \mathrm{CMa}$ by applying the appropriate correction factor interpolated from the tabulation of Davis, Tango, \& Booth (2000). The effective temperature was initially taken to be $6000 \pm 200 \mathrm{~K}$, based on a number of values in the literature, with $\log g=0.6$ and $[\mathrm{Fe} / \mathrm{H}]=0.19$ from Luck \& Lambert $\underline{1985}$ ), for the interpolation. After the effective temperature had been determined to be $5818 \mathrm{~K}$, following the procedure discussed in Section 4 the limb-darkening correction factors were checked using the revised temperature with the same values for $\log g$ and $[\mathrm{Fe} / \mathrm{H}]$. The only change was for $695.6 \mathrm{~nm}$ with an increase from 1.050 to 1.051. Although this has negligible effect, reducing the temperature by only $2 \mathrm{~K}$, the revised value has been used in the final analysis. The correction factors and the resulting values for the limb-darkened angular diameter, based on the values for the uniform-disk angular diameter in Table 1 are listed in Table 2

Table 2: The limb-darkened angular diameter of $\delta$ CMa. $\rho_{\lambda}$ is the ratio of limb-darkened to uniform-disk angular diameter used to convert the uniform-disk angular diameters in Table 1 to the limb-darkened angular diameters in this table (details are given in the text).

\begin{tabular}{cccc}
\hline Instrument & $\begin{array}{c}\lambda \\
(\mathrm{nm})\end{array}$ & $\rho_{\lambda}$ & $\begin{array}{c}\theta_{\mathrm{LD}} \\
(\mathrm{mas})\end{array}$ \\
\hline NSII & 443.0 & 1.099 & $3.62 \pm 0.51$ \\
SUSI & 442.0 & 1.100 & $3.75 \pm 0.11$ \\
SUSI & 442.0 & 1.100 & $3.70 \pm 0.17$ \\
SUSI & 695.6 & 1.051 & $3.633 \pm 0.026$ \\
\hline
\end{tabular}

As noted by Davis et al. (2007) the uncertainty in the NSII value for the limb-darkened angular diam- eter is large and covers all three values determined with SUSI. The new $695.6 \mathrm{~nm}$ value differs from the two $442 \mathrm{~nm}$ values but, although the two $442 \mathrm{~nm}$ values agree with one another, we believe that the new value is the most reliable. The reasons for this belief have been discussed in detail by Davis et al. (2007). In brief, the $442 \mathrm{~nm}$ observations were made during the commissioning phase of SUSI and significant improvements had been made in the observing, calibration and seeing correction techniques prior to the $695.6 \mathrm{~nm}$ observations. In particular, not all the $442 \mathrm{~nm}$ observations of $\delta \mathrm{CMa}$ were bracketed by a calibrator and, as reported by Davis et al. (2007), a re-analysis omitting these data has led to the revised values for the uniformdisk angular diameters listed in Table 1 and, consequently, to the revised values for the limb-darkened angular diameters listed in Table 2 The revised values lie within $\sim 1.1 \sigma$ and $\sim 0.4 \sigma$ of the $695.6 \mathrm{~nm}$ result.

In Table 1 the extrapolated values of $V^{2}$ at zero baseline, $V_{0}^{2}$, for the uniform-disk angular diameter fits to the observational data are listed. The values for the NSII $443 \mathrm{~nm}$ and SUSI $442 \mathrm{~nm}$ observations are all less than the expected value of unity for a single star. The NSII value is consistent with the value for a single star because of its large uncertainty. However, the two SUSI values at $442 \mathrm{~nm}$ are significantly less than unity leading to speculation (Davis et al. 1999b) that $\delta \mathrm{CMa}$ might be a binary system with a significantly fainter companion. As noted by Davis et al. $(1999 \mathrm{~b})$ the fact that the observational points are a reasonable fit to the curve for a single star at $442 \mathrm{~nm}$ suggests that, if the star is a binary system, the $V^{2}$ values at each baseline are averaged over a range in position angles (Hanbury Brown et al. 1967). The $V_{0}^{2}$ values are consistent with a companion 3.25 magnitudes fainter than $\delta \mathrm{CMa}$ at $442 \mathrm{~nm}$. SUSI data at both $442 \mathrm{~nm}$ and $695.6 \mathrm{~nm}$ have been examined for potential position angle variations that would confirm the presence of a companion with a negative result. The value of $V_{0}^{2}$ of $1.003 \pm 0.012$ at $695.6 \mathrm{~nm}$ is consistent with $\delta$ CMa being a single star. The possibility of a faint hot companion significantly affecting the blue measurements while having a negligible effect on the red measurements has been considered. While such a scenario would result in a larger magnitude difference at $700 \mathrm{~nm}$ than at $442 \mathrm{~nm}$ the maximum effect would be a difference of 5 magnitudes resulting in a value 
for $V_{0}^{2}$ of 0.98 . This differs by $\sim 2$ standard deviations from the observed value.

After careful examination of the data and reduction procedures we have concluded that the $695.6 \mathrm{~nm}$ result is correct and that there is no observable companion. The results from the $442 \mathrm{~nm}$ observations must now be regarded as suspect due to the difficulties of calibration and correction of the larger seeing effects at the shorter wavelength. The original agreement between the two $442 \mathrm{~nm}$ results, while encouraging at the time, is thought to be fortuitous. This is supported by the fact that the omission of data not bracketed by a calibrator has resulted in significant changes to the $442 \mathrm{~nm}$ uniform-disk angular diameters and brought them closer to the $695.6 \mathrm{~nm}$ result. The longer wavelength result also has a smaller correction for limb darkening and is therefore less model dependent. For the determination of the bolometric emergent flux and the effective temperature of $\delta \mathrm{CMa}$ we adopt the angular diameter result for $695.6 \mathrm{~nm}$.

\section{The Integrated Flux}

The integrated flux for $\delta$ CMa has been determined following the procedure used by Code et al. (1976) but with a revised estimate for interstellar extinction, improved flux calibrations, and some more recent visual and infrared data. Following Code et al. (1976) it is appropriate to divide the flux measurements into three wavelength regions: ultraviolet, visible and infrared since they rely on different techniques for their calibration. The boundary between the visual and infrared regions has been moved from $810 \mathrm{~nm}$ to $860 \mathrm{~nm}$ due to the availability of new extended visual data and the three regions are discussed individually in Sections 3.2 to 3.4 .

Since $\delta \mathrm{CMa}$ is reddened by interstellar extinction, corrections must be applied in order to determine the emergent flux and effective temperature. This is discussed in the following section.

\subsection{Correction for Interstellar Ex- tinction}

The observed value of $(B-V)$ for $\delta$ CMa is +0.67 (Johnson et 1966) and the intrinsic value for an F8 Iab star is +0.56 (Schmidt-Kaler 1982) giving a colour excess of $E(B-$ $V)$ equal to 0.11 . This is close to the value of 0.12 used by Code et al. (1976) which was based on an intrinsic value of +0.55 by Johnson (1966) but, as pointed out by Fernie (1982), reddenings determined in this way are unreliable because the reddening line so nearly parallels the $(U-B)_{0} \mathrm{v} .(B-V)_{0}$ intrinsic sequence for supergiants. In fact, $\delta \mathrm{CMa}$ lies almost on the intrinsic sequence but closer to G0 than F8.

Feinstein (1967) has studied the young southern cluster Collinder 121 and, from ten early-type mainsequence stars, deduced that $E(B-V)$ for the cluster does not exceed 0.03 . He also associated $\delta$ CMa with the cluster. However, more recent studies (Kaltcheva 2000; Burningham et al. 2007) place Collinder 121 at a distance greater than $1000 \mathrm{pc}$ with a foreground moving association of stars at a distance of $\sim 700 \mathrm{pc}$. With the Hipparcos parallax giving its distance as $550 \pm$ $170 \mathrm{pc}$ it is likely that $\delta \mathrm{CMa}$ is a member of this latter group with $E(B-V)$ of the order of 0.03 . Using spectrum synthesis and model atmospheres Parsons \& Bell (1975) have also derived a value of 0.03 for $E(B-V)$ for $\delta$ CMa. Schmidt (1972) derived a value of 0.05 and McWilliam (1991) used $A_{V}=0.10$, equivalent to $E(B$ $V) \sim 0.03$, derived from 'forcing consistency between all de-reddened colors'.

These alternative approaches to the evaluation of $E(B-V)$ point to a value of $E(B-V)$ of 0.03 and it is clear that the value adopted by Code et al. (1976) is incorrect. We adopt $E(B-V)=0.030$ with an uncertainty of $\sim \pm 0.015$.

The interstellar extinction curve used by Code et al. (1976), and listed in their Table 2, has been adopted to correct the UV fluxes. For the visual and near infrared fluxes $(\lambda \lambda 0.33-1.0 \mu \mathrm{m})$ the average interstellar extinction curve given by Schmidt-Kaler (1982) has been used. For wavelengths in the range $\lambda \lambda 1.0-13.0 \mu \mathrm{m}$ the interstellar extinction law given by Rieke \& Lebofskv (1985) has been adopted. Beyond $13.0 \mu \mathrm{m}$ interstellar extinction is negligible for $\delta$ CMa.

\subsection{The Ultraviolet Flux}

The flux below $330 \mathrm{~nm}$ makes only a small contribution to the total flux $(<1.3 \%)$. We have therefore adopted the flux reported by Code et al. (1976) obtained with the OAO-2 satellite. Application of the revised reddening correction and its uncertainty, as discussed in Section 3.1. gives the flux for the wavelength interval $0-330 \mathrm{~nm}$ equal to $(0.063 \pm 0.013) \times 10^{-9} \mathrm{Wm}^{-2}$.

\subsection{The Visual Flux}

Code et al. (1976) based the visual flux for the wavelength interval $330-810 \mathrm{~nm}$ on the relative spectrophotometric measurements of Davis \& Webb (1974). Subsequently Kiehling (1987) published spectrophotometry for $\delta$ CMa for the wavelength range 325-865 nm. The observations were made at equidistant intervals of $1 \mathrm{~nm}$ with a resolution of $1 \mathrm{~nm}$. The published spectral energy distributions are averaged over band-passes thm wide and are tabulated every $5 \mathrm{~nm}$. The Davis \& Webb (1974) data were published for 25 selected $5 \mathrm{~nm}$ passbands in the wavelength range $330-808 \mathrm{~nm}$. In this section we compare these two sets of data and the empirical MILES fluxes of Sánchez-Blázquez et al. (2006).

Code et al. (1976) used the spectrophotometric calibration of $\alpha$ Lyr (Vega) by Oke \& Schild (1970) to convert the relative spectrophotometry of Davis \& Webb (1974) into a relative absolute flux distribution. Here the more recent spectrophotometric calibration of Vega by Haves (1985) has been used. Following Code et al. (1976) the resulting relative absolute flux distribution has been scaled by the flux ratio corresponding to the monochromatic magnitude of $\delta \mathrm{CMa}$ relative to Vega at $550 \mathrm{~nm}(1.779)$ measured by Davis (private communication). It has then been converted to fluxes using the value for the flux from Vega at $550.0 \mathrm{~nm}$ of 
$3.56 \times 10^{-11} \mathrm{Wm}^{-2} \mathrm{~nm}^{-1}$ (Megessier 1995). The published Kiehling (1987) spectrophotometry is already in the form of a relative absolute flux distribution based on the Hayes calibration. It has been scaled and flux calibrated in exactly the same way as the Davis \& Webb relative absolute flux distribution. The two sets of calibrated flux distributions are in excellent agreement with an RMS difference computed from the wavelengths in common of $<1.1 \%$ with no systematic differences over the wavelength range in common (330$808 \mathrm{~nm}$ ). The two flux distributions are shown in Figure 1.

The flux distribution in the MILES library of empirical spectra (Sánchez-Blázquez et al. 2006) for $\delta \mathrm{CMa}$ has also been considered. Unfortunately the listed MILES fluxes have been de-reddened on the assumption of $E(B-V)=0.209$. The fluxes have been corrected to those for the value of $E(B-V)=0.03$ and calibrated to give absolute fluxes in the same way as the Davis \& Webb and Kiehling data. The wavelength cover of the MILES flux distribution is 355$740 \mathrm{~nm}$, less than the Kiehling range of $325-865 \mathrm{~nm}$. It is tabulated at $0.9 \mathrm{~nm}$ intervals with a resolution of $0.23 \mathrm{~nm}$ compared with the data by Kiehling, which were averaged over $5 \mathrm{~nm}$ intervals and tabulated every $5 \mathrm{~nm}$. To compare the two distributions the fluxes were integrated for the common wavelength range of $355-740 \mathrm{~nm}$. The integrated fluxes agree to within $1 \%$. The difference is small compared with the uncertainty, which is dominated by the uncertainty in the reddening. The Kiehling flux distribution covers a greater wavelength range and extends to the ultraviolet data at the short wavelength end and, for these reasons, it has been used to determine the integrated visual flux.

The visual flux integration has been extended to $860 \mathrm{~nm}$, since Kiehling has data points to $865 \mathrm{~nm}$, rather than terminate it at $810 \mathrm{~nm}$ like Code et al. (1976). Application of the interstellar extinction (reddening) correction to the individual flux values and integration of the resulting dereddened flux distribution, gives the total flux for the wavelength interval $330-860 \mathrm{~nm}$ equal to $(3.05 \pm 0.13) \times 10^{-9} \mathrm{Wm}^{-2}$. The quoted uncertainty is solely due to the uncertainty assigned to $E(B-V)$ but uncertainties in the relative absolute flux distribution of $\delta \mathrm{CMa}$, in the monochromatic magnitude used for scaling, and in the absolute flux calibration must also be considered.

The agreement between all three flux distributions considered, and the good agreement between the integrated fluxes for the wavelength range $355-740 \mathrm{~nm}$ for the MILES and Kiehling flux distributions, suggests that the uncertainty in the relative absolute flux distributions is at the $1 \%$ level. The uncertainty in the monochromatic magnitude difference used for scaling the $\delta \mathrm{CMa}$ flux distribution is estimated to be $\sim 1 \%$ and Megessier (1995) claims $\pm 0.7 \%$ for the flux calibration at $550 \mathrm{~nm}$. The largest uncertainty by far is $\pm 4.3 \%$ due to the uncertainty in $E(B-V)$. The uncertainties are independent and have been combined accordingly to give a resultant uncertainty of $\pm 4.6 \%$. The estimated total flux for the wavelength interval $330-860 \mathrm{~nm}$ is $(3.05 \pm 0.14) \times 10^{-9} \mathrm{Wm}^{-2}$.

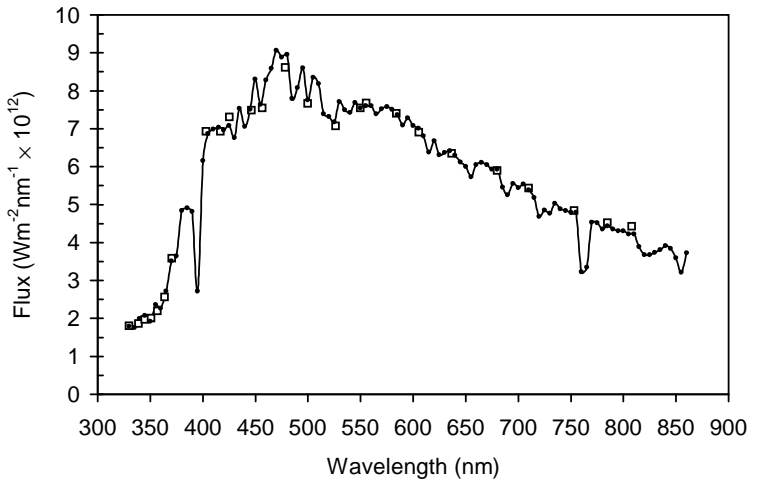

Figure 1: The dereddened visual flux distribution for $\delta$ CMa for the wavelength range $330-860 \mathrm{~nm}$. Key: • (joined by smoothed line) Kiehling (1987); $\square$ Davis \& Webb (1974). Details are given in the text.

\subsection{The Infrared Flux}

More extensive IR data exist than were available to Code et al. (1976), and these have been used to improve the value for the integrated flux in this region. Estimating the total IR flux involves considering data from a number of sources in different forms and with differing calibrations. For this reason the contributions for the wavelength intervals $0.86-1.0 \mu \mathrm{m}, 1.0-2.5 \mu \mathrm{m}$ and $2.5-22.5 \mu \mathrm{m}$ have been considered separately and the results summed. The contribution for wavelengths longer than $22.5 \mu \mathrm{m}$ is negligible $(<0.01 \%$ of the total flux).

Danks \& Dennefeld (1994) give relative spectrophotometry for $\delta \mathrm{CMa}$ for the wavelength range 0.58$1.02 \mu \mathrm{m}$. We have calibrated their data by comparing it with the 7 pass bands of Davis \& Webb (1974) in the overlap region and with the Kiehling (1987) spectrophotometry from $0.58-0.865 \mu \mathrm{m}$. The distributions are in agreement at $0.62 \mu \mathrm{m}$ and the calibration results in a wavelength dependence of $\sim 6.1 \%$ per $100 \mathrm{~nm}$ in the overlap region $0.58-0.86 \mu \mathrm{m}$. This slope correction has been applied to the Danks \& Dennefeld data for the wavelength range $0.58-1.0 \mu \mathrm{m}$. The revised distribution shows good agreement with the $R$ and $I$ broad-band fluxes discussed below and this can be seen in Figure 2. Corrections for reddening have been applied to the resulting flux distribution for the wavelength range $0.86-1.0 \mu \mathrm{m}$ and the flux integrated. The uncertainty in the integrated flux due to the uncertainty in $E(B-V)$ is less than for the $330-860 \mathrm{~nm}$ wavelength range but the uncertainty in the calibration of the fluxes is larger. The overall uncertainty is estimated to be $\pm 3.6 \%$. The resulting estimate for the total flux in the wavelength interval $0.86-1.0 \mu \mathrm{m}$ is $(4.34 \pm 0.16) \times 10^{-10} \mathrm{Wm}^{-2}$.

In the $1-2.5 \mu \mathrm{m}$ interval the only data available are broad-band $J H K$ photometric measurements. Although broad-band IR photometry is not ideally suitable for accurate flux determinations, since it is strongly af- 
fected by atmospheric extinction which changes the effective spectral pass bands in ways that are difficult to take into account (van der Bliek, Manfroid \& Bouchet 1996), we have shown that flux-calibrated RIJHK photometry is consistent with the slope-corrected Danks and Dennefeld flux distribution.

In view of the sparsity of observational data in the 1-2.5 $\mu \mathrm{m}$ interval a model atmosphere flux distribution has been fitted to the dereddened data and used to derive integrated fluxes for this spectral range. The flux in the wavelength range 1.0-2.5 $\mu \mathrm{m}$ has been represented by fitting the flux distribution for a NextGen Model (Hauschildt et al. 1999) to the slope-corrected Danks \& Dennefeld flux distribution plus flux-calibrated $R, I, J, H$ and $K$ broad-band photometry between $0.7 \mu \mathrm{m}$ and $2.2 \mu \mathrm{m}$. The fitted model has a temperature of $5800 \mathrm{~K}(\log g=1.0,[\mathrm{Fe} / \mathrm{H}]=0)$ which is essentially the same as the effective temperature of $5818 \mathrm{~K}$ determined in this work for the star. The photometric magnitudes have been selected and flux calibrated as follows. For $R$ and $I$ the magnitudes by Cousins (1980) have been adopted as they are more reliable than those by Johnson et al. (1966) for these bands (Bessell 2007). They have been calibrated using the absolute flux calibration of Bessell. Castelli \& Plez (1998). For $J, H$ and $K$ the magnitudes were adopted from examination of the photometry of Johnson et al. (1966) ( $J$ and $K)$, Glass (1974) $(J, H$ and $K)$, Engels et al. (1981) $(J, H$ and $K)$ and Carter $(1990)(J, H$ and $K)$. The $J H K$ photometry was flux calibrated using the absolute flux calibrations of both Megessier (1995) and Bessell. Castelli \& Plez (1998). The model flux distribution was fitted by eye to the observational data by means of a scaling factor and the fitted curve and data points are shown in Figure 2 The uncertainty in the integrated flux for the range $1-2.5 \mu \mathrm{m}$ is based on the combination of the estimated uncertainty in the model fit $( \pm 2.5 \%)$, the uncertainty in the absolute flux calibration (taken as $\pm 2 \%$ as given for $J H K$ by Megessier (1995)), and the uncertainty in the dereddening correction $( \pm 1.3 \%)$. The integrated flux for the wavelength range $1.0-2.5 \mu \mathrm{m}$ is $(1.32 \pm 0.05) \times 10^{-9} \mathrm{Wm}^{-2}$.

For the spectrum longward of $2.5 \mu \mathrm{m}$ the $L$ and $M$ photometric bands lie in the $2.5-5.0 \mu \mathrm{m}$ range and there are IRAS Point Source fluxes at 12, 25,60 and $100 \mu \mathrm{m}$ (IRAS Team 1988) and IRAS Low-Resolution Spectra (LRS) covering $~ 7.7-22.7 \mu \mathrm{m}$ (IRAS Team 1986). All these data lie significantly above the fluxes for the model atmosphere fitted to the $0.7-2.2 \mu \mathrm{m}$ interval. Since it is unclear whether the observed flux is from the star or surrounding material we have evaluated the flux longward of $2.5 \mu \mathrm{m}$ in two ways.

Firstly, we have integrated the fitted model fluxes from $2.5-22.5 \mu \mathrm{m}$. The upper wavelength limit corresponds to the long wavelength end of the IRAS LRS spectra. The integrated flux for the $2.5-22.5 \mu \mathrm{m}$ range is $0.164 \times 10^{-9} \mathrm{Wm}^{-2}$.

The second approach has been to use the broadband $L$ and $M$ fluxes, the IRAS Point Source flux at $12 \mu \mathrm{m}$, with the IRAS LRS fluxes and to bridge the gaps in the data by drawing a smooth curve through them. The observational data have been assembled as follows. The magnitudes for the $L$ and $M$ pho-

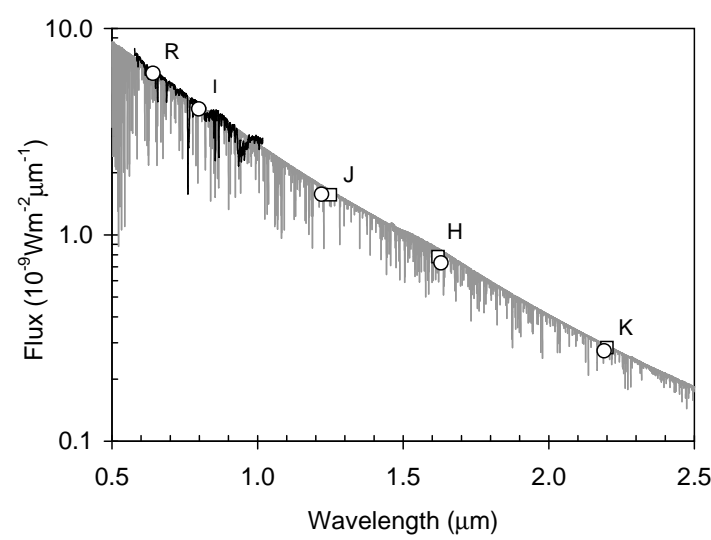

Figure 2: The flux distribution for $\delta \mathrm{CMa}$ for the wavelength range $0.5-2.5 \mu \mathrm{m}$. Key: Black line Danks \& Dennefeld (1994) with slope correction; $\square-R I J H K$ broad-band photometry using absolute flux calibration by Bessell, Castelli \& Plez (1998); ○ - JHK broad-band photometry using absolute flux calibration of Megessier (1995); Gray line fitted model atmosphere. Details are given in the text.

tometric bands have been adopted from examination of the photometry of Johnson et al. (1966) $(L)$, Glass (1974) $(L)$, Engels et al. (1981) $(L$ and $M)$ and Carter (1990) $(L)$. The magnitudes were flux calibrated using the calibration of Megessier (1995) for $L$ and Johnson (1966) for $M$ and corrected for reddening. The IRAS Point Source flux at $12 \mu \mathrm{m}$ was reduced by $4.1 \%$ as proposed by Cohen et al. (1996) to bring it into line with their absolute calibration. The IRAS LRS fluxes have been corrected using the factors determined by Cohen, Walker \& Witteborn (1992) and are claimed to be accurate to better than $2 \%$ (Price et al. 2004). The dereddened and flux calibrated data were plotted against wavelength and a smooth curve drawn through them. The curve was then tabulated at regular intervals across the wavelength range $2.5-22.5 \mu \mathrm{m}$. The $L$ flux lies $\sim 8 \%$ above the model curve, the $M$ flux $\sim 14 \%$ above and the IRAS LRS flux at $8 \mu \mathrm{m} \sim 28 \%$ above. Figure 3 shows the measured flux data, the curve for the model atmosphere flux distribution that was fitted to the wavelength interval $0.7-2.2 \mu \mathrm{m}$, and the smooth curve drawn through the data. The integrated flux in the interval $2.5-22.5 \mu \mathrm{m}$ for the curve drawn through the data is $0.194 \times 10^{-9} \mathrm{Wm}^{-2}$.

The difference in the integrated flux between the two approaches is $\sim 0.03 \times 10^{-9} \mathrm{Wm}^{-2}$. We have adopted the mean value $\left(0.18 \times 10^{-9} \mathrm{Wm}^{-2}\right)$ with an uncertainty of $\pm 0.02 \times 10^{-9} \mathrm{Wm}^{-2}$. This corresponds to an uncertainty of $\pm 0.4 \%$ in the total flux received from the star and translates to an uncertainty of $\pm 0.1 \%$ in the effective temperature $(\sim \pm 6 \mathrm{~K})$.

The total IR flux for the wavelength range 0.86$22.5 \mu \mathrm{m}$ is the sum of the integrated fluxes for the intervals $0.86-1.0 \mu \mathrm{m}, 1.0-2.5 \mu \mathrm{m}, 2.5-22.5 \mu \mathrm{m}$. As noted 


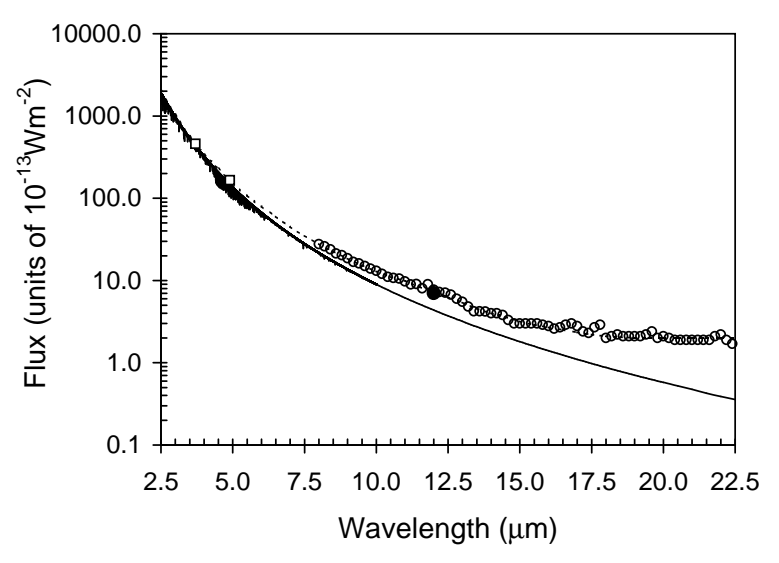

Figure 3: The dereddened flux distribution for $\delta$ CMa for the wavelength range $2.5-22.5 \mu \mathrm{m}$. Key: Solid line - NextGen $5800 \mathrm{~K}$ model; Dashed line - Smooth curve fitted to observational data; $\square$ broad-band $L$ and $M$ fluxes; o - IRAS LRS fluxes; - - IRAS Point Source flux. Details are given in the text.

earlier the flux for wavelengths longer than $22.5 \mu \mathrm{m}$ is negligible. The uncertainty in the total flux was estimated by simply summing the individual errors since they are likely to be dominated by calibration uncertainties and are thus systematic and not independent. The resultant value for the total IR flux is $(1.93 \pm 0.09) \times 10^{-9} \mathrm{Wm}^{-2}$.

\subsection{The Total Flux}

Figure 4 shows the overall extinction-corrected flux distribution, from the visible to the IR, made up of the data used in integrating the visual and IR contributions.

The total flux received from $\delta$ CMa after correction for interstellar extinction is given by summing the contributions from the ultraviolet, visual and infrared regions of the spectrum. The contributions are listed with the total flux of $(5.04 \pm 0.17) \times 10^{-9} \mathrm{Wm}^{-2}$ in Table 3 In assessing the uncertainty in the total flux, the uncertainties from the separate wavelength regions have been considered to be independent.

Table 3: The extinction corrected integrated fluxes for $\delta \mathrm{CMa}$ in each spectral band plus the total integrated flux from the star.

\begin{tabular}{cc}
\hline $\begin{array}{c}\text { Wavelength } \\
(\mathrm{nm})\end{array}$ & $\begin{array}{c}\text { Flux } \\
\left(10^{-9} \mathrm{Wm}^{-2}\right)\end{array}$ \\
\hline $0-330$ & $0.06 \pm 0.01$ \\
$330-860$ & $3.05 \pm 0.14$ \\
$860-\infty$ & $1.93 \pm 0.09$ \\
& \\
Total flux & $5.04 \pm 0.17$ \\
\hline
\end{tabular}

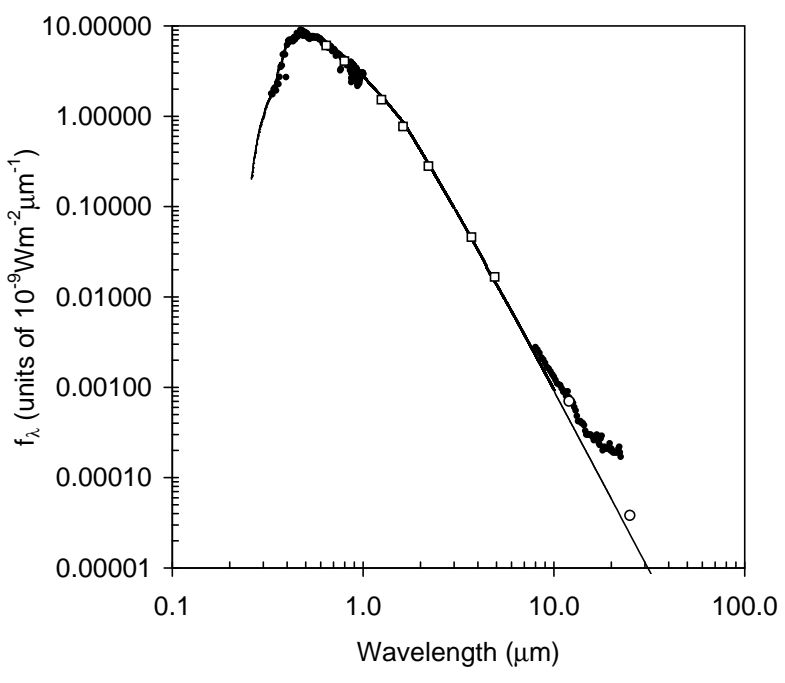

Figure 4: The interstellar extinction-corrected visual and IR flux for $\delta$ CMa. The data are represented in three wavelength ranges by dots: $0.33-0.86 \mu \mathrm{m}$ (Kiehling 1987), 0.86-1.0 $\mu \mathrm{m}$ (Danks \& Dennefeld 1994) and 8.0-22.5 $\mu \mathrm{m}$ IRAS LRS (IRAS Team 1986). $\square$ - fluxes from broadband photometry; $\circ$ - IRAS Point Source fluxes. The line represents the fluxes averaged over $5 \mathrm{~nm}$ bands for the NextGen $5800 \mathrm{~K}$ model atmosphere fitted to the observational data in the $0.7-2.2 \mu \mathrm{m}$ interval. Details of the calibration and integration of the fluxes are given in the text.

The total integrated flux is significantly less than the value of $(6.01 \pm 0.27) \times 10^{-9} \mathrm{Wm}^{-2}$ derived by Code et al. $(1976)$. This is attributable to the revised value for $E(B-V)$. The uncertainty has been reduced due to additional flux measurements in the visual and infrared and improvements in the absolute flux calibration.

\section{The Emergent Flux and Ef- fective Temperature}

The bolometric emergent flux and effective temperature for $\delta \mathrm{CMa}$ are found by substituting the limbdarkened angular diameter and the extinction-corrected total flux received from the star in equation 2 The bolometric emergent flux $\mathcal{F}$ is $(6.50 \pm 0.24) \times 10^{7} \mathrm{Wm}^{-2}$ and the effective temperature $T_{\text {eff }}$ is $5818 \pm 53 \mathrm{~K}$. The dominant source of uncertainty in $T_{\text {eff }}$ is from the integrated flux $(0.8 \%)$, with a smaller contribution from the angular diameter $(0.4 \%)$.

\section{The Radius and Luminosity}

The angular diameter can be combined with the parallax of the star to determine the stellar radius, and the combination of radius and bolometric emergent 
Table 4: The physical parameters determined for $\delta \mathrm{CMa}$.

\begin{tabular}{lc}
\hline \multicolumn{1}{c}{ Parameter } & Value \\
\hline Bolometric emergent & \\
flux $(\mathcal{F})\left(10^{7} \mathrm{Wm}^{-2}\right)$ & $6.50 \pm 0.24$ \\
Effective temperature $\left(T_{\text {eff }}\right)(\mathrm{K})$ & $5818 \pm 53$ \\
Radius $\left(R / R_{\odot}\right)$ & $215 \pm 66$ \\
\hline
\end{tabular}

flux gives the stellar luminosity. Unfortunately the Hipparcos parallax for $\delta \mathrm{CMa}$ is of low accuracy with $\pi=1.82 \pm 0.56$ mas. Nevertheless a value for the radius has been calculated and is listed in Table 4 together with the bolometric emergent flux and effective temperature. The large fractional uncertainty of $\sim \pm 31 \%$ in the parallax dominates the fractional uncertainty in the radius. The luminosity depends on the square of the radius so the percentage error is doubled and the luminosity, with an uncertainty of $\sim \pm 62 \%$, is of little value and has not been listed in Table 4

\section{Discussion}

Previous determinations of the angular diameter, bolometric flux and effective temperature for $\delta$ CMa are listed in Table 5 with the new values presented in this paper. The only other direct determination of effective temperature for this star is by Code et al. (1976) who obtained a value of $T_{\text {eff }}=6110 \pm 430 \mathrm{~K}$ using the angular diameter determined with the NSII. The higher value for the effective temperature determined by Code et al. is almost entirely due to the value of $E(B-V)$ they adopted. While Code et al. underestimated the uncertainty in the bolometric flux by not including an allowance for the uncertainty in the interstellar extinction corrections, the uncertainty in their temperature is primarily due to the uncertainty in the NSII angular diameter. The NSII angular diameter contributed $\pm 6.9 \%$ to the uncertainty in the effective temperature compared with $\pm 1.1 \%$ from the bolometric flux they derived.

The new temperature determination presented here lies within the uncertainty of the Code et al. value but has a substantially reduced uncertainty. The bolometric flux is now the dominant source of uncertainty, primarily due to the interstellar extinction uncertainty in the visible and dependence on broad-band photometry in the near IR.

The infra-red flux method (IRFM) (Blackwell \& Shallis

1977) has been used to determine an angular diameter

and effective temperature for $\delta$ CMa by Blackwell, Petford \& Ahalks nowledgments (1980). Using a value of $(6.0 \pm 0.3) \times 10^{-9} \mathrm{Wm}^{-2}$ for the bolometric flux from the star (Blackwell \& Shallis 1977), they derive an angular diameter of 3.56 mas and an effective temperature of $6143 \mathrm{~K}$. No uncertainty is quoted for the angular diameter, and the effective temperature is suggested to be accurate to about $2 \%$. Their angular diameter only differs from the measured value presented here by $\sim 2.1 \%$ which would only affect the temperature by $\sim 1.1 \%$. The difference in temper-
Table 5: The limb-darkened angular diameter $\theta_{\mathrm{LD}}$, interstellar extinction corrected bolometric flux $f$, and effective temperature $T_{\text {eff for } \delta \mathrm{CMa}}$ from various sources. Key: 1 - Code et al. (1976); 2 - Blackwell. Petford \& Shallis (1980); 3 - McWilliam (1991)); 4 - This work.

\begin{tabular}{clll}
\hline Key & \multicolumn{1}{c}{$\begin{array}{c}\theta_{\mathrm{LD}} \\
(\mathrm{mas})\end{array}$} & $\begin{array}{c}f \\
\left(10^{-9} \mathrm{Wm}^{-2}\right)\end{array}$ & \multicolumn{1}{c}{$\begin{array}{c}T_{\text {eff }} \\
(\mathrm{K})\end{array}$} \\
\hline 1 & $3.60 \pm 0.50$ & $6.01 \pm 0.27$ & $6110 \pm 430$ \\
2 & 3.56 & 6.0 & 6143 \\
3 & & 5.14 & 5855 \\
4 & $3.633 \pm 0.026$ & $5.04 \pm 0.17$ & $5818 \pm 53$ \\
\hline
\end{tabular}

ature of $\sim 5.5 \%$ is mainly due to the larger bolometric flux which is essentially the same as that derived by Code et al. (1976). As discussed in Section 3.1 it is believed that the corrections applied by Code et al. (1976) were too large although it is not known what corrections were applied by Blackwell, Petford \& Shallis (1980).

McWilliam (1991) has also determined an effective temperature for $\delta$ CMa using the IRFM. Using a similar value for the interstellar extinction as in this paper, he derived a value for the bolometric flux of $5.14 \times 10^{-9} \mathrm{Wm}^{-2}$ and an effective temperature of $5855 \mathrm{~K}$. Although no uncertainties were quoted, the value for the effective temperature is a weighted mean of four values, ranging from $5776 \mathrm{~K}$ to $5953 \mathrm{~K}$, each determined from a different IR pass-band. Both the bolometric flux and effective temperature lie within the uncertainties of the new values presented here.

\section{Conclusion}

We have determined new and improved values for the bolometric emergent flux $\left((6.50 \pm 0.24) \times 10^{7} \mathrm{Wm}^{-2}\right)$ and effective temperature $(5818 \pm 53 \mathrm{~K})$ for the $\mathrm{F} 8$ supergiant $\delta \mathrm{CMa}$ using a new interferometric angular diameter measured with SUSI. The uncertainty in the effective temperature has been reduced from $\pm 7.0 \%$ to $\pm 0.9 \%$. It has been shown that precise temperatures can be obtained by combining angular diameters measured interferometrically with bolometric flux distributions assembled from a wide range of sources, with the dominant uncertainty now coming from the bolometric flux determination.

This work has been carried out as part of the SUSI programme which has been funded jointly by the Australian Research Council and the University of Sydney, with additional support from the Pollock Memorial Fund and the Science Foundation for Physics within the University of Sydney. It has made use of the SIMBAD database, operated by CDS, Strasbourg, France and VizieR (Ochsenbein. Bauer \& Marcout 2000). The 
authors are grateful to the referee for a number of suggestions which have improved both the paper and the accuracy of the bolometric flux determination. MJI acknowledges the support of an Australian Postgraduate Award, JRN and APJ the support of University Postgraduate Awards, and APJ and SMO the support of Denison Postgraduate Awards.

\section{References}

Bessell, M. S., Castelli, F., Plez, B. 1998, A\&A, 333, 231

Bessell, M. S. 2007, private communication

Blackwell, D. E., Shallis, M. J. 1977, MNRAS, 180, 177

Blackwell, D. E., Petford, A. D., Shallis, M. J. 1980, A\&A, 82, 249

van der Bliek, N. S., Manfroid, J., Bouchet, P. 1996, A\&A Supp, 119, 547

Burningham, B., Naylor, T., Jeffries, R. D., Devey, C. R. 2007 MNRAS, in press

Carter, B. S. 1990,MNRAS, 242, 1

Code, A. D., Davis, J., Bless, R. C., Hanbury Brown R. 1976, ApJ, 203, 417

Cohen, M., Walker, R. G., Witteborn, F. C. 1992, AJ, 104, 2030

Cohen, M., Witteborn, F. C., Carbon, D. F., Davies, J. K., Wooden, D. H., Bregman, J. D. 1996, AJ, 112,2274

Cousins, A. W. 1980, SAAO Circ. 1, 234

Danks, A. C., Dennefeld, M. 1994, PASP, 106, 382

Davis, J. et al. 2007, PASA, 24, in press, page number to come.

Davis, J., Tango, W. J., Booth, A. J. 2000, MNRAS, 318,387

Davis, J., Tango, W. J., Booth, A. J., ten Brummelaar, T. A., Minard, R. A., Owens, S. M. 1999a, MNRAS, 303, 773

Davis, J., Tango, W. J., Booth, A. J., Thorvaldson, E. D., Giovannis, J. 1999b, MNRAS, 303, 783

Davis, J., Webb, R. J. 1974, MNRAS, 168, 163

Engels, D., Sherwood, W. A., Wamsteker, W., Schultz, G. V. 1981, A\&A Supp., 45, 5

Feinstein, A. 1967, ApJ, 149, 107

Fernie, J. D. 1982, ApJ, 257, 193

Glass, I. S. 1974, MNASSA, 33, 53

Hanbury Brown, R., Davis, J., Allen L. R., Rome, J. M. 1967, MNRAS, 137, 393

Hanbury Brown, R., Davis, J., Allen L. R. 1974, MNRAS, 167, 121

Hauschildt, P. H., Allard, F., Ferguson, J., Baron, E., Alaxander, D. R. 1999, ApJ, 525, 871

Hayes, D. S. 1985, in IAU Symp. 111, Calibration of Fundamental Stellar Quantities, eds. D. S. Hayes, L. E. Pasinetti, A. G. Davis Philip, (Dordrecht, Reidel), 225

IRAS Team 1987, IRAS Low Resolution Spectra, NASA RP-1190

IRAS Team 1988, The IRAS Point Source Catalogue, version 2.0, NASA RP-1190

Johnson, H. L. 1966, ARA\&A, 4, 193

Johnson, H. L., Mitchell, R. I., Iriarte, B., Wisniewski,

W. Z. 1966, Comm. Lunar and Plan. Lab., 4, 99

Kaltcheva, N. T. 2000, MNRAS, 318, 1023

Kiehling, R. 1987, A\&AS, 69, 465

Luck, R. E., Lambert, D. L. 1985, ApJ, 298, 782

McWilliam, A. 1991, AJ, 101, 1065

Megessier, C. 1995, A\&A, 296, 771

Oke, J. B., Schild, R. E. 1970, ApJ, 161, 1015

Ochsenbein, F., Bauer, P., Marcout, J. 2000, A\&AS, 143, 221

Parsons, S. B., Bell, R. A. 1975, in Dudley Observatory Report No. 9, Multicolor Photometry and the Theoretical HR Diagram, eds. A. G. Davis Philip, D. S. Hayes (Dudley Observatory, Albany, New York), 73

Price, S. D., Paxson, C., Engelke, C., Murdock, T. L. 2004, AJ, 128, 889

Rieke, G. H., Lebofsky, M. J. 1985, ApJ, 288, 618

Sánchez-Blázquez et al. 2006, MNRAS, 371, 703

Schmidt, E. G. 1972, ApJ, 174, 595

Schmidt-Kaler, Th. 1982, in Landolt-Bornstein (New Series) Vol. 3, eds. K. Schaifers, H. H. Voigt (Berlin: Springer-Verlag), 1 